\title{
Tecnologías del aprendizaje y conocimiento en el desarrollo de las competencias cognitivas en estudiantes universitarios
}

\author{
Learning technologies and knowledge in the development of cognitive competencies in university \\ students
}

Aprender tecnologias e conhecimentos no desenvolvimento de competências cognitivas em estudantes universitários

\author{
Juana Victoria Bustinza Vargas \\ jbustinza@unap.edu.pe \\ https://orcid.org/0000-0003-4081-5733 \\ Universidad Nacional del Altiplano, Perú
}

\author{
Lourdes Lacuta Sapacayo \\ llacuta@unap.edu.pe \\ https://orcid.org/0000-0002-3315-6330 \\ Universidad Nacional del Altiplano, Perú
}

\section{RESUMEN}

Estudiar la Influencia de las Tecnologías de aprendizaje-conocimiento en el desarrollo de las competencias cognitivas; implica analizar desde una perspectiva educacional; el objetivo general es determinar la influencia de las Tecnologías del Aprendizaje y Conocimiento (TAC) en el proceso del desarrollo de las competencias cognitivas en estudiantes de la Escuela Profesional de Trabajo Social-UNA-Puno2019-I. El enfoque es cuantitativo, método hipotético deductivo. Tipo de investigación básico teórico de nivel correlacionalcausal, diseño no experimental de corte transversal. La muestra es 242 estudiantes del I al X semestre, el instrumento aplicado es el cuestionario, prueba estadística Chi cuadrado de Pearson.El resultado es que existe influencia significativa entre las dos variables, el docente al utilizar el aula virtual logra mayor escala académica a diferencia de los que no la utilizan. La conclusión es que al utilizar aulas virtuales se mejora el proceso de aprendizaje, logrando la interactualidad de conocimientos a nivel mundial.

Palabras clave: Tecnología de aprendizaje y conocimiento, estudiantes universitarios

\section{ABSTRACT}

Study the influence of learning-knowledge technologies in the development of cognitive skills; It implies analyzing from an educational perspective; The general objective is to determine the influence of Learning and Knowledge Technologies (TAC) in the process of the development of cognitive competences in students of the Professional School of Social Work-UNAPuno-2019-I. The approach is quantitative, hypothetical deductive method. Type of theoretical basic research of correlationalcausal level, non-experimental crosssectional design. The sample is 242 students from I to X semester, the applied instrument is the questionnaire, Pearson's Chi-square statistical test. The result is that there is significant influence between the two variables, the teacher when using the virtual classroom achieves a higher academic scale than of those who do not use it. The conclusion is that by using virtual classrooms, the learning process is improved, achieving the interaction of knowledge worldwide.

Key words: Learning and knowledge technology, university students

\section{RESUMO}

Estudar a influência das tecnologias de aprendizagem-conhecimento no desenvolvimento de habilidades cognitivas; implica analisar a partir de uma perspectiva educacional; $\mathrm{O}$ objetivo geral é determinar a influência das Tecnologias de Aprendizagem e Conhecimento (TAC) no processo de desenvolvimento de competências cognitivas em alunos da Escola Profissional de Trabalho SocialUNA-Puno-2019-I. A abordagem é quantitativa, método dedutivo hipotético. Tipo de pesquisa teórica básica de nível correlacional-causal, delineamento transversal não experimental. A amostra é de 242 alunos do I ao X semestre, o instrumento aplicado é o questionário, teste estatístico do Qui-quadrado de Pearson, o resultado é que há influência significativa entre as duas variáveis, o professor ao utilizar a sala de aula virtual atinge uma escala acadêmica superior daqueles que não o usam. Conclui-se que, com a utilização de salas de aula virtuais, o processo de aprendizagem é aprimorado, alcançando a interação do conhecimento em todo o mundo.

Palavras-chave: Aprendizagem e tecnologia do conhecimento, estudantes universitários 


\section{INTRODUCCIÓN}

El tema de la tecnología de la información y el conocimiento fue introducido veinte años después de popularizarse la noción de la sociedad postindustrial, en la década de los ochenta del siglo pasado emergiendo en los países industrializados más avanzados estas nuevas tecnologías denominadas TIC, ya conocida como sociedad del conocimiento (Castells, 2001) a un nuevo paradigma tecnológico.

Para el caso dela educación, las TAC contribuyen no sólo al aprendizaje individualizado sino de forma planificada y orientada por el docente, se produce un aprendizaje en colectivo. Es un hecho conocido y documentado la gran brecha que existe entre el sistema educativo, metodologías y maestros, frente a un alumnado denominado hoy en día "nativos digitales" (Chávez, 2015) en un mundo dónde las tecnologías digitales y virtuales hacen parte del día a día de la mayoría de la población. Así pues, el reto de estimular el uso de las tecnologías del aprendizaje-conocimiento, es una tarea de grandes proporciones que debe preocupar, tanto a los entes gubernamentales en términos de políticas públicas educativas, como al sistema escolar en general, a las instituciones de educación superior y a los maestros en particular, siempre y cuando que se emplee de manera debida. En el Perú nos encontramos regido por la SUNEDU con la ley Universitaria 30220 en la que nos plantea el desarrollo de la educación desde las competencias, complementando con las tecnologías, para desarrollar estudiantes desde un enfoque socio-crítico, y ello comprende en el artículo Nro. 47 (Ley Universitaria, 2014) donde nos indica que las universidades pueden desarrollar programas de educación a distancia, basados en entornos virtuales de aprendizaje, donde debemos de desarrollar las habilidades necesarias para su emprendimiento y aplicación en la Universidad Nacional del Altiplano, regido al perfil básico del egresado de dicha universidad, específicamente a las competencias genéricas en cuanto al uso de las tecnologías de la información y comunicación.

El motivo de esta investigación es determinar la influencia de las Tecnologías del Aprendizaje y Conocimiento (TAC) en el proceso del desarrollo de las competencias cognitivas en estudiantes de la Escuela Profesional de Trabajo Social-UNA-Puno2019-I. El propósito es mostrar como las tecnologías del aprendizaje y conocimiento se vienen aplicando en la Facultad de Trabajo Social y su influencia en las competencias cognitivas de los estudiantes, a través de la implementación de programas web 2.0 y la utilización de herramientas virtuales en relación con la competencia cognitiva que hoy se analiza desde el enfoque socio-crítico.

\section{MÉTODO}

La investigación fue realizada bajo el enfoque cuantitativo, tuvo por método el hipotético deductivo. El tipo de investigación es básico teórico de nivel correlacional-causal, diseño no experimental de corte transversal. La población está determinada por 652 estudiantes y la muestra ascendió a 242 estudiantes entre varones y mujeres de la Facultad de Trabajo Social distribuidos del I al X semestre académico 2019-I, los instrumentos que permitieron recoger la información necesaria respecto a las variables de estudio Tecnología del aprendizaje conocimiento y proceso de aprendizaje, aplicado a los estudiantes de la escuela profesional de Trabajo Social-UNA-Puno 2019 I fueron la 
escala de Likert a 5 escalas, con un total de 17 ítems distribuidos en: variable $\mathrm{x}-14$ ítems y variable $\mathrm{y}$ - 3 ítems, como segundo instrumento escala nominal con 22 ítems siendo de la variable $\mathrm{x}-15$ ítems y de la variable y-07 ítems. Y como prueba estadística Chi cuadrado de Pearson con el programa SPPS versión 24.

\section{RESULTADOS}

Se dará a conocer según las dimensiones de la
En la Tabla 1 se puede observar en cuanto al uso de las tecnologías del aprendizaje conocimiento según el proceso de desarrollo de las competencias cognitivas de los estudiantes; del total de 242 estudiantes el mayor porcentaje se centra en un $32.2 \%$ viene hacer 78 estudiantes su proceso de aprendizaje es regular. Seguido del $22.8 \%$ que representa a 55 estudiantes manifiestan que el uso de las tecnologías es casi siempre siendo el proceso de aprendizaje bueno. investigación como son:

Tabla 1. Uso de las tecnologías del aprendizaje conocimiento según proceso de del desarrollo de las competencias cognitivas de los estudiantes.

\begin{tabular}{|c|c|c|c|c|c|c|c|c|c|c|}
\hline \multirow{3}{*}{$\begin{array}{l}\text { Uso de las Tecnologías } \\
\text { del Aprendizaje } \\
\text { conocimiento }\end{array}$} & \multicolumn{10}{|c|}{ Proceso de desarrollo de las competencias cognitivas de los estudiantes } \\
\hline & \multicolumn{2}{|c|}{ Buenas } & \multicolumn{2}{|c|}{ Regulares } & \multicolumn{2}{|c|}{ Malas } & \multicolumn{2}{|c|}{$\mathbf{N} / \mathbf{S}$} & \multicolumn{2}{|c|}{ Total } \\
\hline & $\mathbf{N}$ & $\%$ & $\mathbf{N}$ & $\%$ & $\mathbf{N}$ & $\%$ & $\mathbf{N}$ & $\%$ & $\mathbf{N}$ & $\%$ \\
\hline Siempre & 3 & $1.2 \%$ & 2 & $0.8 \%$ & 0 & $0.0 \%$ & 1 & $0.4 \%$ & 6 & $2.4 \%$ \\
\hline Casi siempre & 55 & $22.8 \%$ & 42 & $17.4 \%$ & 0 & $0.0 \%$ & 0 & $0.0 \%$ & 97 & $40.1 \%$ \\
\hline Casi nunca & 25 & $10.3 \%$ & 78 & $32.2 \%$ & 0 & $0.0 \%$ & 1 & $0.4 \%$ & 104 & $43.0 \%$ \\
\hline Nunca & 17 & $7.1 \%$ & 16 & $6.6 \%$ & 2 & $0.8 \%$ & 0 & $0.0 \%$ & 35 & $14.5 \%$ \\
\hline Total & 100 & $41.4 \%$ & 138 & $57.0 \%$ & 2 & $0.8 \%$ & 2 & $0.8 \%$ & 242 & $100.0 \%$ \\
\hline
\end{tabular}

Fuente: Encuesta aplicada por la alumna maestrante de Trabajo Social Mención Gestión de Recursos Humanos a los estudiantes de 1ro a 10mo semestre de la escuela profesional de Trabajo Social-UNA Puno 2019 I.

\section{Prueba de hipótesis general}

Ha: Las Tecnologías del Aprendizaje y Conocimiento (TAC) influyen en el proceso de desarrollo de las competencias cognitivas de los estudiantes de la Escuela Profesional de Trabajo Social-UNA-Puno-2019-I.
Ho: Las Tecnologías del Aprendizaje y Conocimiento (TAC) no influyen en el proceso de desarrollo de las competencias cognitivas de los estudiantes de la Escuela Profesional de Trabajo Social-UNA-Puno-2019-I. 
Tabla 2. Prueba de hipótesis general.

\begin{tabular}{lccc}
\hline & Valor & Significación asintótica (bilateral) \\
\hline Chi-cuadrado de Pearson & $119,318^{\mathrm{a}}$ & 2 & 0,000 \\
Razón de verosimilitud & 142,872 & 2 & 0,000 \\
Asociación lineal por Lineal & 4,137 & 1 & 0,042 \\
N de casos válidos & 242 & & \\
\hline
\end{tabular}

Según la prueba de chi cuadrado se pudo determinar que existe influencia entre el uso de las Tecnologías del Aprendizaje conocimiento en el proceso de desarrollo de las competencias cognitivas de los estudiantes de la escuela Profesional de Trabajo Social - UNA-2019-I, puesto que demuestra con evidencia estadística.

Tomando un valor de $\mathrm{x}^{2}$ es 119,318 con 2 grados de libertad (gl), con una significancia de
0.000 puesto que esta probabilidad es menor a 0.05 por lo tanto, se rechaza la hipótesis nula y se acepta la hipótesis alterna, afirmando que existe influencia significativa entre las dos variables. Con nivel de confianza equivalente a un $95 \%$ por lo cual podemos decir que la hipótesis es válida (Tabla 2).

Tabla 3. Nivel de integración de las TAC en las diversas asignaturas según número de materias cursadas en el semestre I 2019 aprobadas por los estudiantes.

\begin{tabular}{|c|c|c|c|c|c|c|c|c|}
\hline \multirow{3}{*}{$\begin{array}{c}\text { Nivel de integración de las TAC en las } \\
\text { diversas asignaturas }\end{array}$} & \multicolumn{8}{|c|}{ Nro de materias cursadas en el semestre I 2019 aprobadas por los estudiantes } \\
\hline & \multicolumn{2}{|c|}{ 1-3 } & \multicolumn{2}{|c|}{ 4-6 } & \multicolumn{2}{|c|}{ 7- a más } & \multicolumn{2}{|c|}{ Total } \\
\hline & $\mathbf{N}$ & $\%$ & $\mathbf{N}$ & $\%$ & $\mathbf{N}$ & $\%$ & $\mathbf{N}$ & $\%$ \\
\hline Escaso nivel de integración & 1 & $0.4 \%$ & 55 & $22.7 \%$ & 39 & $16.1 \%$ & 95 & $39.3 \%$ \\
\hline Parcialmente integrado en la asignaturas & 0 & $0.0 \%$ & 45 & $18.6 \%$ & 89 & $36.8 \%$ & 134 & $55.4 \%$ \\
\hline Bien integrado en las asignaturas & 0 & $0.0 \%$ & 3 & $1.2 \%$ & 10 & $4.1 \%$ & 13 & $5.4 \%$ \\
\hline Total & 1 & $0.4 \%$ & 103 & $42.6 \%$ & 138 & $57.0 \%$ & 242 & $100.0 \%$ \\
\hline
\end{tabular}

Fuente: Encuesta aplicada por la alumna maestrante de Trabajo Social Mención Gestión de Recursos Humanos a los estudiantes de 1ro a 10mo semestre de la escuela profesional de Trabajo Social-UNA Puno 2019 I.

En la Tabla 3 en relación de Nivel de integración de las TAC en las diversas asignaturas según el Número de materias cursadas en el semestre I-2019 aprobadas por los estudiantes se tiene en mayor porcentaje un nivel de integración parcialmente de las TAC en las diversas asignaturas en un $36.8 \%$ en relación al número de materias cursadas aprobadas de 7 a más seguida del escaso nivel de integración en un $22.7 \%$ en relación a 4-6 cursos aprobados. 
Tabla 4. Pruebas de chi-cuadrado.

\begin{tabular}{llcc}
\hline & \multicolumn{2}{c}{ Pruebas de chi-cuadrado } & \\
& Valor & Gl & Significación asintótica (bilateral) \\
\hline Chi-cuadrado de Pearson & $46,310^{\mathrm{a}}$ & 30 & 0.029 \\
Razón de verosimilitud & 35.171 & 30 & 0.236 \\
Asociación lineal por Lineal & 0.497 & 1 & 0.481 \\
N de casos válidos & 242 & & \\
\hline
\end{tabular}

Considerando la prueba estadística de la Chicuadrado de Pearson para la Tabla 3 en relación al nivel de integración de las TAC en las diversas asignaturas y el número de materias cursadas en el semestre I 2019 aprobadas por los estudiantes de la escuela profesional de Trabajo Social UNA-Puno
2019 I, hacemos la referencia que los resultados son: el nivel de significancia tiene el valor de $\boldsymbol{\alpha}=$ $\mathbf{0 . 0 5}$ y el valor obtenido es de $\mathbf{0 . 0 2 9}$; entonces es menor al nivel de significancia, lo que significa que se acepta la $\mathbf{H} \mathbf{1}$ (hipótesis alterna) y se rechaza la $\mathbf{H}_{\mathbf{0}}$ (hipótesis nula).

Tabla 5. Tipo de aula virtual que utilizan tus docentes con mayor frecuencia según total de materias cursadas 2019 I aprobadas.

\begin{tabular}{|c|c|c|c|c|c|c|c|c|}
\hline \multirow{3}{*}{$\begin{array}{l}\text { Tipo de aula virtual utilizan tus } \\
\text { docentes con mayor frecuencia }\end{array}$} & \multicolumn{8}{|c|}{ Total de materias cursadas 2019 I logro aprobar } \\
\hline & \multicolumn{2}{|c|}{$1-3$} & \multicolumn{2}{|c|}{ 4-6 } & \multicolumn{2}{|c|}{7 a más } & \multicolumn{2}{|c|}{ Total } \\
\hline & $\mathbf{N}$ & $\%$ & $\mathbf{N}$ & $\%$ & $\mathbf{N}$ & $\%$ & $\mathbf{N}$ & $\%$ \\
\hline Classroom & 1 & $0.4 \%$ & 48 & $19.8 \%$ & 28 & $11.6 \%$ & 77 & $31.8 \%$ \\
\hline Aula Virtual UNA-Puno & 0 & $0.0 \%$ & 4 & $1.7 \%$ & 8 & $3.3 \%$ & 12 & $5.0 \%$ \\
\hline Blackboard & 0 & $0.0 \%$ & 22 & $9.1 \%$ & 30 & $12.4 \%$ & 52 & $21.5 \%$ \\
\hline Ninguno & 2 & $0.8 \%$ & 41 & $16.9 \%$ & 58 & $24.0 \%$ & 101 & $41.7 \%$ \\
\hline Total & 3 & $1.2 \%$ & 115 & $47.5 \%$ & 124 & $51.2 \%$ & 242 & $100.0 \%$ \\
\hline
\end{tabular}

Fuente: Encuesta aplicada por la alumna maestrante de Trabajo Social Mención Gestión de Recursos Humanos a los estudiantes de 1ro a 10mo semestre de la escuela profesional de Trabajo Social-UNA Puno 2019 I.

Como se puede apreciar en la Tabla 5 en relación al tipo de aula virtual que utilizan los docentes con mayor frecuencia, encontramos ninguno, en mayor porcentaje con un $24 \%$ en relación al total de materias cursadas 2019 I aprobadas que fueron de 7 a más.
Y en segundo dato de importancia el tipo de aula virtual que utilizan los docentes con mayor frecuencia es el classroom, en un $19.8 \%$ en relación al total de materias cursadas 2019 I aprobadas fueron de 4-6. 
Tabla 6. Pruebas de chi - cuadrado.

\begin{tabular}{llll}
\hline & \multicolumn{2}{c}{ Pruebas de chi-cuadrado } & \\
& Valor & Gl & Significación asintótica (bilateral) \\
\hline Chi-cuadrado de Pearson & $85,994 \mathrm{a}$ & 20 & 0.029 \\
Razón de verosimilitud & 24.357 & 20 & 0.236 \\
Asociación lineal por Lineal & 4.046 & 1 & 0.481 \\
N de casos válidos & 242 & & \\
\hline
\end{tabular}

Considerando la prueba estadística de la Chicuadrado de Pearson para la Tabla 5 en relación al tipo de aula virtual que utilizan los docentes con mayor frecuencia y el total de materias cursadas 2019 I aprobadas por los estudiantes de la escuela profesional de Trabajo Social UNA-Puno 2019 I, hacemos la referencia que los resultados son de: El nivel de significancia tiene el valor de $\boldsymbol{\alpha}=\mathbf{0 . 0 5} \mathrm{y}$ el valor obtenido es de $\mathbf{0 . 0 0 0}$; entonces es menor al nivel de significancia, lo que significa que se acepta la $\mathbf{H} \mathbf{1}$ (hipótesis alterna) y se rechaza la $\mathbf{H}_{\mathbf{0}}$ (hipótesis nula).

\section{Discusión}

En Vega (2017) estudio realizado en Lima, llega a la conclusión que el Valor $\mathrm{p}=0.005<0.05$, afirmando en un $95 \%$ existe la probabilidad de una influencia significativa entre el uso de las tecnología de la información y comunicación con la enseñanza aprendizaje del idioma inglés en los estudiantes del I y II ciclo de la Escuela Académica Profesional de la facultad de educación de la Universidad Nacional Mayor de San Marcos he ahí la importancia del uso de las TAC en los procesos de enseñanza de los estudiantes universitarios y estar preparados tanto docentes como estudiantes en un contexto donde las tecnologías vienen hacer protagonistas; lo cual coincide con esta investigación, destacando que el uso de las Tecnologías del aprendizaje conocimiento realmente influyen en el proceso del desarrollo de las competencias cognitivas; como se puede ver, en el proceso cuanto más es el uso de las TAC mejor son los resultados de aprendizaje.

Para Nuñez (2016) sobre la administración de las tecnologías de información y comunicación y el proceso de aprendizaje en el sistema de educación a distancia en el Perú incide positivamente en la satisfacción de las necesidades de los estudiantes eliminando restricciones de distancia o de tiempo. A diferencia de la investigación no se ve un impacto significativo porque la enseñanza es exclusivamente presencial no dando la importancia a las TAC, pero en el caso que habría la necesidad de implementar una enseñanza a distancia se tendría dificultades; porque se demuestra que la plana docente $y$ estudiantes aún están en proceso de familiarizarse con el uso de las TAC.

\section{CONCLUSIONES}

Es así que se comprueba que al utilizar aulas virtuales las calificaciones de los estudiantes es mayor mejorando su proceso de aprendizaje formando así estudiantes con un pensamiento crítico y con un conocimiento amplio debido al 
acceso y la interactualidad de conocimientos a nivel mundial con el uso de las TAC.

Se puede mencionar que la limitante para el uso de las TAC es que los estudiantes no cuentan con aulas con acceso a wi-fi más aun en el caso de Trabajo Social que cuenta con dos pabellones, uno antiguo y otro nuevo, este último no cuenta con internet por ser construcción nueva. Y para el uso de las TAC es necesario el acceso al internet, convirtiéndose este en una limitante principal, este servicio debería ser proporcionado por la universidad ya que los estudiantes en su mayoría son de provincia y de escasos recursos económicos.

Se puede mencionar en relación a los datos que está en proceso el uso de las aulas virtuales y que para los estudiantes existe una mejor aprensión de conocimiento con el uso de tecnologías ya quea través de videos, uso de bibliotecas virtuales, WhatsApp, y otros existe una mejor interconectividad que permite mejorar sus conocimientos. Es ahí la importancia de incluir en el proceso de enseñanza la tecnología para lograr un mejor nivel de coeficiente intelectual en los estudiantes, ello también les servirá para su vida profesional ya que en toda área de intervención esta inmiscuida la tecnología y no podemos estar ajenas a ello.

\section{REFERENCIAS}

Castells, M. (2001). La era de la información: vol. 3 fin del milenio; Editorial Alianza; http://saber. ucab.edu.ve/handle/123456789/33417

Chávez, M. A. (2015). Cómo enseñar a las nuevas generaciones digitales. Revista Electrónica de Investigación Educativa, 17(2), 1-4. Recuperado de http://redie.uabc.mx/vol17no2/contenidocanor.html

Ley 30220/2014 (9 de julio). Ley Universitaria. Diario Oficial el Peruano No 12914- 527211

Nuñez, F. (2016). La administración de las tecnologías de la información y comunicación y el proceso de aprendizaje en el sistema de educación a distancia en el Perú. (Tesis de maestría). Universidad Inca Garcilaso de la Vega. Perú

Vega, C. (2017). Uso de las TICS y su influencia con la enseñanza - aprendizaje del idioma inglés en los estudiantes del I y II ciclo de la Escuela Académico Profesional de la Facultad de Educación UNMSM-Lima. (Tesis de maestría). Universidad Nacional de San Marcos. Lima, Perú 